\title{
General results on the decomposition of transitive fuzzy relations
}

\author{
Susana Díaz Bernard De Baets Susana Montes
}

\begin{abstract}
We study the transitivity of fuzzy preference relations, often considered as a fundamental property providing coherence to a decision process. We consider the transitivity of fuzzy relations w.r.t. conjunctors, a general class of binary operations on the unit interval encompassing the class of triangular norms usually considered for this purpose. Having fixed the transitivity of a large preference relation w.r.t. such a conjunctor, we investigate the transitivity of the strict preference and indifference relations of any fuzzy preference structure generated from this large preference relation by means of an (indifference) generator. This study leads to the discovery of two families of conjunctors providing a full characterization of this transitivity. Although the expressions of these conjunctors appear to be quite cumbersome, they reduce to more readily used analytical expressions when we focus our attention on the particular case when the transitivity of the large preference relation is expressed w.r.t. one of the three basic triangular norms (the minimum, the product and the Eukasiewicz triangular norm) while at the same time the generator used for decomposing this large preference relation is also one of these triangular norms. During our discourse, we pay ample attention to the Frank family of triangular norms/copulas.
\end{abstract}

keywords Fuzzy preference relation Transitivity Conjunctor Indifference generator Frank family of t-norms

\section{Introduction}

The pairwise comparison of possible alternatives is a first step in many approaches to decision making. If this first step lacks coherence, the whole decision process might become meaningless. A popular criterion for coherence is the transitivity of preferences, expressing that the strength of the link between two alternatives cannot be weaker than the strength of any chain involving another alternative. Along this paper, we will consider a decision agent with a certain decision policy, which will be used to compare a finite set of alternatives $A$. In classical preference modelling (see e.g. $[4,32]$ ), there exists a basic relation $R$ on $A$, called large preference relation, such that for any two alternatives $a$ and $b,(a, b) \in R$ expresses that alternative $a$ is considered to be at least as good as 
alternative $b$. This relation $R$ is further decomposed into a strict preference relation $P$, an indifference relation $I$ and an incomparability relation $J$. It is well known that the transitivity of $R$ is completely characterized by the transitivity of $P$ and $I$ and two additional transitivity-like inequalities involving $P$ and $I$. In case the relation $R$ is complete, i.e. any two alternatives are comparable, the transitivity of $R$ is fully characterized by the transitivity of $P$ and $I$ (see [4]).

The relations $R, P$ and $I$ play a central role in decision making and are used frequently in the social sciences. However, as these relations are crisp, they are not always appropriate to model human decisions. This lack of flexibility has led to the introduction of fuzzy relations. They are often used as alternative to crisp relations as they allow the decision maker to express a degree of preference rather than its presence or absence only. Since then, fuzzy preference relations have been widely studied and applied (see e.g. [5, 20, 23, 31]). A key notion in this context is the notion of a fuzzy preference structure (see e.g. [5, 10, 21, 22, $26,38]$ ); see [9] for a historical account of its development.

Not surprisingly, in this fuzzy context transitivity is again one of the most important properties (see e.g. [5, 23, 27]). Traditionally, transitivity of a fuzzy relation is defined w.r.t. a triangular norm. In this setting, the transitivity of the large preference relation $R$ has been characterized in a similar way as in the crisp case, however, for strongly complete $R$ only [14]. However, strong completeness is a rather restrictive condition, since even not every fuzzy preference structure with an empty incomparability relation fulfills it. Subsequent works have therefore treated more general large preference relations [6, 7]. These works concentrate on the propagation of a fixed type of transitivity from a large preference relation $R$ to various associated strict preference $(P)$ and indifference (I) relations. We continue along this line of research, but treat the problem from a much more general viewpoint. First, we work with conjunctors, a class of operations broader than the class of triangular norms; hence, we consider also more general types of transitivity. Second, we consider all possible strict preference and indifference relations associated with a large preference relation, making explicit use of (indifference) generators to decompose a large preference relation. Third, we do not just study the possible preservation of the transitivity when decomposing $R$, but we identify the strongest types of transitivity we can assure for the $P$ and $I$. In preceding work [19], we have fenced in the types of transitivity of $P$ and $I$ depending upon the type of transitivity $R$ satisfies and the generator used for constructing them. Here, we take another leap forward: we provide an explicit expression for the conjunctor characterizing the transitivity that any $I$ (resp. $P$ ) satisfies, provided it is generated by means of a given generator from a large preference relation $R$ satisfying a given type of transitivity. We prove that no stronger type of transitivity is fulfilled by all such $I$ (resp. $P$ ). In such case, we will speak of a strongest result possible. This means that the conjunctor we will present is such that all $I$ (resp. $P$ ), satisfy the transitivity w.r.t. that conjunctor, and that for any operation greater than that conjunctor, we can provide at least one large preference relation $R$ such that the associated $I$ (resp. $P$ ), does not satisfy the transitivity w.r.t. that greater operation. Obviously, this does not prevent the existence of some individual 
large preference relation (such as a crisp one) for which the associated $I$ (resp. $P)$ satisfies a stronger type of transitivity.

This work is structured as follows. In Section 2 we recall the most relevant concepts concerning (fuzzy) preference structures. In Section 3 we discuss conjunctors and related operations, and some of their useful properties. In Section 4 we characterize the transitivity of the indifference relation $I$ by means of a general conjunctor and simplify the general expression for the most important particular cases. In Section 5 we characterize the transitivity of the strict preference relation $P$ by means of another general conjunctor, and give ample attention to the most important particular cases. Concluding remarks and further considerations are given in Section 6 .

\section{Preference structures}

\subsection{Crisp preference structures}

Consider a decision maker who is presented a set of alternatives $A$. Let us suppose that this person compares the alternatives two by two. Given two alternatives, the decision maker can act in one of the following three ways: (i) he/she clearly prefers one to the other; (ii) the two alternatives are indifferent to him/her; (iii) he/she is unable to compare the two alternatives. According to these cases, we can define three (binary) relations on $A$ : the strict preference relation $P$, the indifference relation $I$ and the incomparability relation $J$. Thus, for any $(a, b) \in A^{2}$, we classify:

$$
\begin{array}{lll}
(a, b) \in P & \Leftrightarrow & \text { he/she prefers } a \text { to } b \\
(a, b) \in I & \Leftrightarrow & a \text { and } b \text { are indifferent to him/her; } \\
(a, b) \in J & \Leftrightarrow & \text { he/she is unable to compare } a \text { and } b .
\end{array}
$$

We recall that for a relation $Q$ on $A$, its converse is defined as $Q^{t}=\{(b, a) \mid$ $(a, b) \in Q\}$, its complement as $Q^{c}=\{(a, b) \mid(a, b) \notin Q\}$ and its dual as $Q^{d}=\left(Q^{t}\right)^{c}$. One easily verifies that $P, I, J$ and $P^{t}$ establish a particular partition of $A^{2}[32]$.

Definition 2.1 A preference structure on $A$ is a triplet $(P, I, J)$ of relations on A that satisfies:

(i) $P$ is irreflexive, $I$ is reflexive and $J$ is irreflexive;

(ii) $P$ is asymmetrical, $I$ and $J$ are symmetrical;

(iii) $P \cap I=\emptyset, P \cap J=\emptyset$ and $I \cap J=\emptyset$;

(iv) $P \cup P^{t} \cup I \cup J=A^{2}$.

A preference structure $(P, I, J)$ on $A$ is characterized by the reflexive relation $R=P \cup I$, called large preference relation, in the following way:

$$
(P, I, J)=\left(R \cap R^{d}, R \cap R^{t}, R^{c} \cap R^{d}\right) .
$$


Conversely, for any reflexive relation $R$ on $A$, the triplet $(P, I, J)$ constructed in this way from $R$ is a preference structure on $A$ such that $R=P \cup I$. As $R$ is the union of the strict preference relation and the indifference relation, $(a, b) \in R$ means that $a$ is at least as good as $b$.

A relation $Q$ on $A$ is called transitive if $((a, b) \in Q \wedge(b, c) \in Q) \Rightarrow(a, c) \in Q$, for any $(a, b, c) \in A^{3}$. Recall that the composition of two relations $Q_{1}$ and $Q_{2}$ on $A$ is the relation $Q_{1} \circ Q_{2}$ on $A$ defined as $Q_{1} \circ Q_{2}=\{(a, b) \mid(\exists c \in A)((a, c) \in$ $\left.\left.Q_{1} \wedge(c, b) \in Q_{2}\right)\right\}$. It is clear that $Q$ is transitive if and only if $Q \circ Q \subseteq Q$. The transitivity of the large preference relation $R$ can be characterized as follows [4].

Theorem 2.1 For any reflexive relation $R$ with associated preference structure $(P, I, J)$ it holds that

$$
R \circ R \subseteq R \Leftrightarrow(P \circ P \subseteq P \wedge I \circ I \subseteq I \wedge P \circ I \subseteq P \wedge I \circ P \subseteq P) .
$$

In case $R$ is complete, i.e. $R \cup R^{t}=A^{2}$, this characterization can be simplified as follows. Note that the completeness of $R$ is equivalent to stating that any two elements are comparable, i.e. $J=\emptyset$.

Theorem 2.2 For any complete reflexive relation $R$ with corresponding preference structure $(P, I, \emptyset)$ it holds that

$$
R \circ R \subseteq R \Leftrightarrow(P \circ P \subseteq P \wedge I \circ I \subseteq I) .
$$

Finally, we recall an important characterization of preference structures. Let us identify relations with their characteristic mapping, then Definition 2.1 can be written in the following minimal way [13]: $I$ is reflexive and symmetrical, and for any $(a, b) \in A^{2}$ it holds that

$$
P(a, b)+P^{t}(a, b)+I(a, b)+J(a, b)=1 .
$$

Classical, also called crisp, preference structures can therefore also be considered as Boolean preference structures, employing 1 and 0 for describing presence or absence of strict preference, indifference and incomparability.

\subsection{Additive fuzzy preference structures}

A serious drawback of classical preference structures is their inability to express intensities. In contrast, in fuzzy preference modelling, strict preference, indifference and incomparability are a matter of degree. These degrees can take any value in the unit interval $[0,1]$ and fuzzy relations are used for capturing them [22].

The intersection of fuzzy relations is defined pointwisely based on some triangular norm (t-norm for short), i.e. an increasing, commutative and associative binary operation on $[0,1]$ with neutral element 1 . The three most important t-norms are the minimum operator $T_{\mathbf{M}}(x, y)=\min (x, y)$, the algebraic product $T_{\mathbf{P}}(x, y)=x \cdot y$ and the Eukasiewicz t-norm $T_{\mathbf{L}}(x, y)=\max (x+y-1,0)$. 
Another important t-norm is the drastic product defined by

$$
T_{\mathbf{D}}(x, y)= \begin{cases}\min (x, y) & , \text { if } \max (x, y)=1, \\ 0 & , \text { otherwise }\end{cases}
$$

According to the usual ordering of functions, the above t-norms can be ordered as follows: $T_{\mathbf{D}} \leq T_{\mathbf{L}} \leq T_{\mathbf{P}} \leq T_{\mathbf{M}}$. In fact, the greatest t-norm is the minimum operator and the smallest t-norm is the drastic product. Another important t-norm in the study of the propagation of transitivity is the nilpotent minimum $T_{\text {nM }}$ defined by

$$
T_{\mathbf{n M}}(x, y)= \begin{cases}\min (x, y) & , \text { if } x+y>1, \\ 0 & , \text { otherwise }\end{cases}
$$

Similarly, the union of fuzzy relations is based on a t-conorm, i.e. an increasing, commutative and associative binary operation on $[0,1]$ with neutral element 0 . T-norms and t-conorms come in dual pairs: to any t-norm $T$ there corresponds a t-conorm $S$ through the relationship $S(x, y)=1-T(1-x, 1-y)$. For the above three t-norms, we thus obtain the maximum operator $S_{\mathbf{M}}(x, y)=\max (x, y)$, the probabilistic sum $S_{\mathbf{P}}(x, y)=x+y-x y$ and the Eukasiewicz t-conorm (bounded sum) $S_{\mathbf{L}}(x, y)=\min (x+y, 1)$. For more background on t-norms and t-conorms and the notations used in this paper, we refer to [29].

The definition of a fuzzy preference structure has been a topic of debate during several years (see e.g. $[22,37,38])$. Accepting the assignment principle - for any pair of alternatives $(a, b)$ the decision maker is allowed to assign at least one of the degrees $P(a, b), P(b, a), I(a, b)$ and $J(a, b)$ freely in the unit interval - has finally led to a fuzzy version of Definition 2.1 with intersection based on the Łukasiewicz t-norm and union based on the Lukasiewicz t-conorm. Interestingly, a corresponding minimal definition is identical to the classical one provided we replace crisp relations by fuzzy relations: a triplet $(P, I, J)$ of fuzzy relations on $A$ is a fuzzy preference structure on $A$ if and only if $I$ is reflexive and symmetrical, and for any $(a, b) \in A^{2}$ it holds that

$$
P(a, b)+P^{t}(a, b)+I(a, b)+J(a, b)=1,
$$

where $P^{t}(a, b)=P(b, a)$. This identity explains the name additive fuzzy preference structures.

Another topic of controversy has been how to construct such a fuzzy preference structure from a reflexive fuzzy relation. Alsina [1] proved a kind of impossibility theorem showing that a construction based on a single t-norm is unfeasible. As a reaction, Fodor and Roubens adopted an axiomatic approach [22]. The most recent and most successful approach is that of De Baets and Fodor based on (indifference) generators [10].

Definition 2.2 A generator $i$ is a commutative binary operation on the unit interval $[0,1]$ that is bounded by the Eukasiewicz t-norm $T_{\mathbf{L}}$ and the minimum operator $T_{\mathbf{M}}$, i.e. $T_{\mathbf{L}} \leq i \leq T_{\mathbf{M}}$. 
Note that generators are not necessarily t-norms, albeit having neutral element 1. With a given generator $i$, we associate the following binary operations on $[0,1]$ :

$$
\begin{aligned}
& p(x, y)=x-i(x, y), \\
& j(x, y)=i(x, y)-(x+y-1) .
\end{aligned}
$$

The triplet $(p, i, j)$ is called a generator triplet. For any reflexive fuzzy relation $R$ on $A$ it holds that the triplet $(P, I, J)$ of fuzzy relations on $A$ defined by:

$$
\begin{aligned}
P(a, b) & =p(R(a, b), R(b, a))=R(a, b)-i(R(a, b), R(b, a)), \\
I(a, b) & =i(R(a, b), R(b, a)), \\
J(a, b) & =j(R(a, b), R(b, a))=i(R(a, b), R(b, a))-(R(a, b)+R(b, a)-1),
\end{aligned}
$$

is an additive fuzzy preference structure on $A$ such that $R=P \cup_{S_{\mathrm{L}}} I$, i.e. $R(a, b)=P(a, b)+I(a, b)$.

Note that the definition of a generator does not mention monotonicity. In accordance with the monotonicity axiom of Fodor and Roubens [22], a generator triplet $(p, i, j)$ is called monotone if:

(i) $p$ is increasing in the first and decreasing in the second argument;

(ii) $i$ is increasing in both arguments;

(iii) $j$ is decreasing in both arguments.

Recall that a binary operation $C:[0,1]^{2} \rightarrow[0,1]$ is called a quasi-copula [24] if it is increasing, has neutral element 1 and is 1-Lipschitz continuous, i.e.

$$
\left|C\left(x_{1}, y_{1}\right)-C\left(x_{2}, y_{2}\right)\right| \leq\left|x_{1}-x_{2}\right|+\left|y_{1}-y_{2}\right|,
$$

for any $\left(x_{1}, x_{2}, y_{1}, y_{2}\right) \in[0,1]^{4}$. For any quasi-copula $C$ it holds that $T_{\mathbf{L}} \leq C \leq$ $T_{\mathrm{M}}$. Quasi-copulas were introduced by Alsina et al. [3] as a weaker variant of the well-known copulas in probability theory [30]. Recall that a binary operation $C:[0,1]^{2} \rightarrow[0,1]$ is called a copula if it has absorbing element 0 , neutral element 1 and is 2 -increasing, i.e.

$$
C\left(x_{1}, y_{1}\right)+C\left(x_{2}, y_{2}\right) \geq C\left(x_{1}, y_{2}\right)+C\left(x_{2}, y_{1}\right),
$$

for all $\left(x_{1}, x_{2}, y_{1}, y_{2}\right) \in[0,1]^{4}$ such that $x_{1} \leq x_{2}$ and $y_{1} \leq y_{2}$.

Nowadays quasi-copulas are witnessing increasing popularity in fuzzy logic (see e.g. [11, 28]). In some applications, the defining property of 1-Lipschitz continuity proves to be more decisive than the associativity of t-norms. The following theorem characterizes monotone generator triplets and points out the importance of quasi-copulas [10].

Theorem 2.3 A generator triplet $(p, i, j)$ is monotone if and only if $i$ is a commutative quasi-copula. 
The most popular generators are undeniably the Frank t-norms (see e.g. [22]). In [10] it was proven that when using a generator $i$ that is a t-norm, then also $p(x, 1-y)$ is a t-norm if and only if $i$ is a Frank t-norm; in that case, $p(x, 1-y)$ and $j(1-x, 1-y)$ are also Frank t-norms. Moreover, the Frank t-norms are also copulas (and therefore quasi-copulas). For the sake of completeness, we recall that the Frank t-norms are given by

$$
T_{\lambda}^{\mathbf{F}}(x, y)= \begin{cases}T_{\mathbf{M}}(x, y) & , \text { if } \lambda=0, \\ T_{\mathbf{P}}(x, y) & \text { if } \lambda=1, \\ T_{\mathbf{L}}(x, y) & \text { if } \lambda=\infty \\ \log _{\lambda}\left(1+\frac{\left(\lambda^{x}-1\right)\left(\lambda^{y}-1\right)}{\lambda-1}\right) & , \text { otherwise. }\end{cases}
$$

For any $\lambda \in[0, \infty]$ and any $(x, y) \in[0,1]^{2}$, it holds that

$$
T_{1 / \lambda}^{\mathbf{F}}(x, y)=x-T_{\lambda}^{\mathbf{F}}(x, 1-y) .
$$

Moreover, for any $\lambda \in] 0, \infty\left[\right.$, it holds that $T_{\lambda}^{\mathbf{F}}(x, y)=\phi_{\lambda}^{-1}\left(\phi_{\lambda}(x) \phi_{\lambda}(y)\right)$, with $\phi_{1}(x)=x$ and $\phi_{\lambda}(x)=\frac{\lambda^{x}-1}{\lambda-1}$ for $\left.\lambda \in\right] 0,1[\cup] 1, \infty[$.

\section{Conjunctors}

\subsection{Generalizing $T$-transitivity}

The usual way of defining the transitivity of a fuzzy relation is w.r.t. a t-norm $T$ : a fuzzy relation $Q$ on $A$ is called $T$-transitive if $T(Q(a, b), Q(b, c)) \leq Q(a, c)$ for any $(a, b, c) \in A^{3}$. However, the restriction to t-norms is questionable. On the one hand, even when the large preference relation $R$ is $T$-transitive w.r.t. a t-norm $T$, the transitivity of the generated $P$ and $I$ cannot always be expressed w.r.t. a t-norm $[17,18,19]$. On the other hand, the results presented in the following sections also hold when $R$ is transitive w.r.t. a more general operation. From the point of view of fuzzy preference modelling, it is not that surprising that the class of t-norms is too restrictive, as a similar conclusion was drawn when identifying suitable generators, as was briefly explained in the previous section. There, continuity, in casu the 1-Lipschitz property, was more important than associativity. As discussed in $[18,19]$, suitable operations for defining the transitivity of fuzzy relations are conjunctors.

Definition 3.1 A conjunctor $f$ is an increasing binary operation on $[0,1]$ that coincides on $\{0,1\}^{2}$ with the Boolean conjunction.

The smallest conjunctor $c_{S}$ and greatest conjunctor $c_{G}$ are given by

$$
c_{S}(x, y)= \begin{cases}0, & \text { if } \min (x, y)<1 \\ 1, & \text { otherwise }\end{cases}
$$

and

$$
c_{G}(x, y)= \begin{cases}0, & \text { if } \min (x, y)=0 \\ 1, & \text { otherwise }\end{cases}
$$


Obviously, $c_{S} \leq T_{\mathbf{D}} \leq T_{\mathbf{M}} \leq c_{G}$.

Given a conjunctor $f$, we say that a fuzzy relation $Q$ on $A$ is $f$-transitive if $f(Q(a, b), Q(b, c)) \leq Q(a, c)$ for any $(a, b, c) \in A^{3}$. Clearly, for two conjunctors $f$ and $g$ such that $f \leq g$, it holds that $g$-transitivity implies $f$-transitivity. Restricting our attention to reflexive fuzzy relations only, such as large preference relations, not all conjunctors are suitable for defining transitivity. Indeed, for a reflexive fuzzy relation $R$ it holds that

$$
\begin{aligned}
& f(R(a, b), R(c, d)) \leq f(R(a, b), 1)=f(R(a, b), R(b, b)) \leq R(a, b), \\
& f(R(a, b), R(c, d)) \leq f(1, R(c, d))=f(R(c, c), R(c, d)) \leq R(c, d),
\end{aligned}
$$

and thus $f(R(a, b), R(c, d)) \leq \min (R(a, b), R(c, d))$. Hence, for reflexive fuzzy relations, we should consider conjunctors upper bounded by $T_{\mathrm{M}}$ only.

Defining the composition $Q_{1} \circ_{f} Q_{2}$ w.r.t. a conjunctor $f$ of two fuzzy relations $Q_{1}$ and $Q_{2}$ on $A$ by

$$
Q_{1} \circ_{f} Q_{2}(a, c)=\sup _{b} f\left(Q_{1}(a, b), Q_{2}(b, c)\right),
$$

still allows us to use the shorthand $Q \circ_{f} Q \subseteq Q$ to denote $f$-transitivity.

\subsection{Dominance and bisymmetry}

The dominance relation is a well-known relation on the class of t-norms (see e.g. $[29,34])$ and its usefulness has been demonstrated several times (see e.g. [12, 35]). It can be straightforwardly generalized to conjunctors [33].

Definition 3.2 A conjunctor $f_{1}$ is said to dominate a conjunctor $f_{2}$, denoted $f_{1} \gg f_{2}$, if for any $(x, y, z, t) \in[0,1]^{4}$ it holds that

$$
f_{1}\left(f_{2}(x, y), f_{2}(z, t)\right) \geq f_{2}\left(f_{1}(x, z), f_{1}(y, t)\right) .
$$

Every t-norm is dominated by itself and by the greatest t-norm, the minimum operator. It also holds that if a t-norm $T_{1}$ dominates another t-norm $T_{2}$, then $T_{1} \geq T_{2}$. Conjunctors behave differently than t-norms. For instance, not every conjunctor dominates itself [19]. Also, for conjunctors, the dominance relation is not related to the usual ordering of binary operations. For instance, the minimum operator (which is not the greatest conjunctor), dominates any other conjunctor [19]. The notion of self-dominance of conjunctors is obviously equivalent the well-known property of bisymmetry (see e.g. [29]).

Definition 3.3 A conjunctor $f$ is said to be bisymmetric if for any $(x, y, z, t) \in$ $[0,1]^{4}$ it holds that

$$
f(f(x, y), f(z, t))=f(f(x, z), f(y, t)) .
$$

Every associative and commutative binary operation on $[0,1]$ is bisymmetric. Also, a bisymmetric binary operation on $[0,1]$ with neutral element 1 is associative and commutative. For more details on this property we refer to [2]. 


\subsection{Fuzzy implications and related operations}

With a given t-norm $T$, one usually associates a fuzzy implication (also called $R$-implication or $T$-residuum) as a binary operation on $[0,1]$ defined by (see e.g. $[22,29])$ :

$$
\mathcal{I}_{T}(x, y)=\sup \{z \in[0,1] \mid T(x, z) \leq y\} .
$$

When $T$ is left-continuous it holds that $T(x, z) \leq y \Leftrightarrow z \leq \mathcal{I}_{T}(x, y)$, and $\mathcal{I}_{T}$ is called the residual implicator of $T$. In this paper, we associate two binary operations with any commutative conjunctor.

Definition 3.4 With a given commutative conjunctor $f$ we associate two binary operations $\mathcal{I}_{f}$ and $\mathcal{J}_{f}$ on the unit interval defined by

$$
\begin{aligned}
& \mathcal{I}_{f}(x, y)=\sup \{z \in[0,1] \mid f(x, z) \leq y\} \\
& \mathcal{J}_{f}(x, y)=\inf \{z \in[0,1] \mid f(x, z) \geq y\}
\end{aligned}
$$

The above definition could also be extended to non-commutative operations, but in that case we should distinguish between left and right operations. In this work we will only consider the case of commutative operations (commutative conjunctors or generators).

Clearly, $\mathcal{I}_{f}$ and $\mathcal{J}_{f}$ are decreasing in their first argument and increasing in their second argument. Under a mild condition, the operation $\mathcal{I}_{f}$ has an interesting logical interpretation; the operation $\mathcal{J}_{f}$, however, does not admit such an interpretation.

Definition 3.5 An implicator $f$ is a binary operation on $[0,1]$ that is decreasing in its first argument, increasing in its second argument and that coincides on $\{0,1\}^{2}$ with the Boolean implication.

Proposition 3.1 Consider a commutative conjunctor $f$, then $\mathcal{I}_{f}$ is an implicator if and only if $f(1, y)>0$, for any $y>0$.

The condition in the preceding proposition is obviously fulfilled when $f$ has 1 as neutral element. 
Lemma 3.2 Consider a commutative conjunctor $f$. Then it holds that:

(i) If $f$ is left-continuous, then

$$
f(x, z) \leq y \quad \Leftrightarrow \quad z \leq \mathcal{I}_{f}(x, y) .
$$

(ii) If $f$ is upper bounded by $T_{\mathbf{M}}$, then

$$
x \leq y \quad \Rightarrow \quad \mathcal{I}_{f}(x, y)=1 .
$$

(iii) If $f$ is left-continuous and has 1 as neutral element, then

$$
x \leq y \quad \Leftrightarrow \quad \mathcal{I}_{f}(x, y)=1 .
$$

(iv) If $f$ is continuous and has 1 as neutral element, then

$$
y \leq x \quad \Rightarrow \quad f\left(x, \mathcal{I}_{f}(x, y)\right)=y .
$$

Other properties of residual implications of left-continuous t-norms can be found, for example, in [29].

\section{Transitivity of indifference relations}

\subsection{Main result}

This section is dedicated to indifference relations. As shown in Subsection 2.2, given a generator $i$ and a large preference relation $R$, the indifference relation $I$ is defined as $I=i\left(R, R^{t}\right)$. We study the minimal transitivity guaranteed for $I$ when we fix the transitivity of $R$ w.r.t. a conjunctor $h$. We start by recalling some upper and lower bounds for the transitivity of $I$ [19]. First, we know that $I$ is at least $c_{S}$-transitive when $R$ is $h$-transitive, irrespective of the conjunctor $h$. Second, the transitivity of $I$ is upper bounded by $\min (h, i)$ transitivity. However, these are only upper and lower bounds. Next, we provide a full characterization of the transitivity of $I$. We only need to require the increasingness of the generator $i$, a very mild condition.

Theorem 4.1 Consider an increasing generator $i$ and a conjunctor $h$. For any reflexive fuzzy relation $R$ with corresponding indifference relation I generated by means of $i$, it holds that

$$
R \text { is h-transitive } \Rightarrow I \text { is } f_{h}^{i} \text {-transitive, }
$$

where $f_{h}^{i}$ is the conjunctor defined by

$$
f_{h}^{i}(x, y)=\inf _{\substack{1 \geq u \geq x \\ 1 \geq v \geq y}} i\left(h(u, v), h\left(\mathcal{J}_{i}(v, y), \mathcal{J}_{i}(u, x)\right)\right) .
$$

Moreover, if $i$ is right-continuous, this is the strongest result possible. 
Proof. First, we prove that $f_{h}^{i}$ is a conjunctor. Obviously, $f_{h}^{i}$ is a binary operation on $[0,1]$. Since $i$ has neutral element 1 , it holds that $\mathcal{J}_{i}(1,1)=1$ and

$$
f_{h}^{i}(1,1)=i\left(h(1,1), h\left(\mathcal{J}_{i}(1,1), \mathcal{J}_{i}(1,1)\right)\right)=i(1,1)=1 .
$$

Further, considering $(u, v)=(x, y)$, for $(1,0),(0,1)$ and $(0,0)$, we have

$$
\begin{aligned}
& f_{h}^{i}(1,0) \leq i\left(h(1,0), h\left(\mathcal{J}_{i}(0,0), \mathcal{J}_{i}(1,1)\right)\right)=0, \\
& f_{h}^{i}(0,1) \leq i\left(h(0,1), h\left(\mathcal{J}_{i}(1,1), \mathcal{J}_{i}(0,0)\right)\right)=0, \\
& f_{h}^{i}(0,0) \leq i\left(h(0,0), h\left(\mathcal{J}_{i}(0,0), \mathcal{J}_{i}(0,0)\right)\right)=0,
\end{aligned}
$$

whence $f_{h}^{i}(1,0)=f_{h}^{i}(0,1)=f_{h}^{i}(0,0)=0$. On the other hand, $f_{h}^{i}$ has to be increasing. Let us consider $x_{1} \leq x_{2}$ and $y_{1} \leq y_{2}$. For all $(u, v) \geq\left(x_{2}, y_{2}\right)$, it holds that $\mathcal{J}_{i}\left(u, x_{1}\right) \leq \mathcal{J}_{i}\left(u, x_{2}\right)$ and $\mathcal{J}_{i}\left(v, y_{1}\right) \leq \mathcal{J}_{i}\left(v, y_{2}\right)$, whence

$$
i\left(h(u, v), h\left(\mathcal{J}_{i}\left(v, y_{1}\right), \mathcal{J}_{i}\left(u, x_{1}\right)\right)\right) \leq i\left(h(u, v), h\left(\mathcal{J}_{i}\left(v, y_{2}\right), \mathcal{J}_{i}\left(u, x_{2}\right)\right)\right) .
$$

Hence, $f_{h}^{i}\left(x_{1}, y_{1}\right) \leq f_{h}^{i}\left(x_{2}, y_{2}\right)$ and $f_{h}^{i}$ is a conjunctor.

Second, we prove the implication. It suffices to prove that for any $(a, b, c) \in$ $A^{3}$ it holds that $f_{h}^{i}(I(a, b), I(b, c)) \leq I(a, c)$. By definition, it holds that $I(a, b)=$ $i(R(a, b), R(b, a))$ and $I(b, c)=i(R(b, c), R(c, b))$. Hence,

$$
\begin{aligned}
& R(b, a) \geq \inf \{z \in[0,1] \mid i(R(a, b), z)=I(a, b)\}=\mathcal{J}_{i}(R(a, b), I(a, b)) \\
& R(c, b) \geq \inf \{t \in[0,1] \mid i(R(b, c), t)=I(b, c)\}=\mathcal{J}_{i}(R(b, c), I(b, c)) .
\end{aligned}
$$

From the $h$-transitivity of $R$ and the monotonicity of $i$ it follows that

$$
\begin{aligned}
& I(a, c)=i(R(a, c), R(c, a)) \\
& \geq i(h(R(a, b), R(b, c)), h(R(c, b), R(b, a))) \\
& \geq i\left(h(R(a, b), R(b, c)), h\left(\mathcal{J}_{i}(R(b, c), I(b, c)), \mathcal{J}_{i}(R(a, b), I(a, b))\right)\right) \\
& \geq f_{h}^{i}(I(a, b), I(b, c)) .
\end{aligned}
$$

Finally, we prove that no greater conjunctor qualifies when $i$ is right-continuous. Consider a conjunctor $g>f_{h}^{i}$, then we need to prove that there exists a reflexive fuzzy relation $R$ that is $h$-transitive, but for which the corresponding indifference relation is not $g$-transitive. Since $g>f_{h}^{i}$, there exists $\left(x_{0}, y_{0}\right) \in[0,1]^{2}$ such that $g\left(x_{0}, y_{0}\right)>f_{h}^{i}\left(x_{0}, y_{0}\right)$. For $(x, y) \in[0,1]^{2}$, let us denote

$$
F_{h}^{i}(u, v)=i\left(h(u, v), h\left(\mathcal{J}_{i}(v, y), \mathcal{J}_{i}(u, x)\right)\right),
$$

then

$$
f_{h}^{i}(x, y)=\inf _{\substack{1 \geq u \geq x \\ 1 \geq v \geq y}} F_{h}^{i}(u, v) .
$$

Since $\epsilon=g\left(x_{0}, y_{0}\right)-f_{h}^{i}\left(x_{0}, y_{0}\right)>0$, there exists $u_{0} \geq x_{0}$ and $v_{0} \geq y_{0}$ such that

$$
F_{h}^{i}\left(u_{0}, v_{0}\right)<f_{h}^{i}\left(x_{0}, y_{0}\right)+\epsilon=g\left(x_{0}, y_{0}\right) .
$$


Let us call $z_{0}=\min \left\{z \in[0,1] \mid i\left(u_{0}, z\right)=x_{0}\right\}$ and $t_{0}=\min \left\{t \in[0,1] \mid i\left(v_{0}, t\right)=\right.$ $\left.y_{0}\right\}$. Since $i$ is right-continuous, it holds that $i\left(u_{0}, z_{0}\right)=x_{0}$ and $i\left(v_{0}, t_{0}\right)=y_{0}$. The reflexive fuzzy relation $R$ on $A=\{a, b, c\}$ given by

\begin{tabular}{c|ccc}
$R$ & $a$ & $b$ & $c$ \\
\hline$a$ & 1 & $u_{0}$ & $h\left(u_{0}, v_{0}\right)$ \\
$b$ & $z_{0}$ & 1 & $v_{0}$ \\
$c$ & $h\left(t_{0}, z_{0}\right)$ & $t_{0}$ & 1
\end{tabular}

is $h$-transitive, but the corresponding indifference relation $I$ generated by means of $i$ is not $g$-transitive. Indeed, $I$ is given by

\begin{tabular}{c|ccc}
$I$ & $a$ & $b$ & $c$ \\
\hline$a$ & 1 & $x_{0}$ & $F_{h}^{i}\left(u_{0}, v_{0}\right)$ \\
$b$ & $x_{0}$ & 1 & $y_{0}$ \\
$c$ & $F_{h}^{i}\left(u_{0}, v_{0}\right)$ & $y_{0}$ & 1
\end{tabular}

and

$$
I(a, c)=F_{h}^{i}\left(u_{0}, v_{0}\right)<g\left(x_{0}, y_{0}\right)=g(I(a, b), I(b, c)) .
$$

Remark 4.1 Note that the monotonicity of the generator $i$ is necessary to ensure that the binary operation $f_{h}^{i}$ is a conjunctor. Indeed, consider the generator $i$ defined by

$$
i(x, y)= \begin{cases}0 & , \text { if } \min (x, y)>0.3 \text { and } \max (x, y)<0.4, \\ T_{\mathbf{M}}(x, y) & , \text { otherwise },\end{cases}
$$

and the conjunctor $h=T_{\mathbf{P}}$. It holds that

$$
\begin{aligned}
& f_{h}^{i}(0.5,0.5)=\inf _{\substack{1 \geq u \geq 0.5 \\
1 \geq v \geq 0.5 \\
1 \geq 0}} i(u \cdot v, 0.25)=0.25, \\
& f_{h}^{i}(0.6,0.6)=\inf _{\substack{1 \geq u \geq 0.6 \\
1 \geq v \geq 0.6}} i(u \cdot v, 0.36)=0 .
\end{aligned}
$$

Hence, $f_{h}^{i}$ is not increasing, and is therefore not a conjunctor.

Remark 4.2 Note also that the right-continuity of the generator $i$ is necessary to ensure that the binary operation $f_{h}^{i}$ defines the strongest result possible. Indeed, consider the increasing generator $i$ defined by

$$
i(x, y)= \begin{cases}0 & , \text { if }(\min (x, y)<0.2 \text { and } x+y \leq 1) \\ & \text { or }(\min (x, y)=0.2 \text { and } \max (x, y) \leq 0.4) \\ 0.2 & , \text { if }(\min (x, y)=0.2 \text { and } \max (x, y)>0.4) \\ & \text { or }(\min (x, y) \in] 0.2,0.8] \text { and } x+y \leq 1) \\ 0.4 & , \text { if } \min (x, y) \in[0.4,0.6] \text { and } \max (x, y) \leq 0.7 \\ & \text { and } x+y>1, \\ 0.6 & , \text { if } x, y \in] 0.6,0.7] \\ \min (x, y) & , \text { otherwise, }\end{cases}
$$


and the conjunctor $h=T_{\mathbf{L}}$. For any $T_{\mathbf{L}}$-transitive reflexive fuzzy relation $R$, it follows from Theorem 4.1 that the corresponding indifference relation I generated by means of $i$ is $f_{h}^{i}$-transitive. Note that $i$ is not right-continuous.

Let us now consider the binary operation $f^{*}$ on $[0,1]$ defined by $f^{*}(x, y)=$ $f_{h}^{i}(x, y)$ if $\min (x, y)<0.6$, and $f^{*}(x, y)=\max \left(f_{h}^{i}(x, y), 0.2\right)$ otherwise. It is clear that $f^{*} \geq f_{h}^{i}$. Moreover, it holds that $f_{h}^{i}(0.6,0.6) \leq F_{h}^{i}(0.65,0.65)=0$, whence $f_{h}^{i}(0.6,0.6)=0$. Thus, $f^{*}$ is strictly greater than $f_{h}^{i}$ at least in the point $(0.6,0.6)$.

To prove that $I$ is also $f^{*}$-transitive, it suffices to show that for any $(a, b, c) \in$ $A^{3}$ such that $\min (I(a, b), I(b, c)) \geq 0.6$, it holds that $I(a, c) \geq 0.2$. We will use the notation $x=I(a, b), y=I(b, c), u=R(a, b), v=R(b, c), z=R(b, a)$ and $t=R(c, b)$. Then, $x=i(u, z)$ and $y=i(v, t)$. Since $R$ is $h$-transitive, it holds that $R(a, c) \geq h(u, v)$ and $R(c, a) \geq h(z, t)$. Further, since $i$ is increasing, we have $I(a, c)=i(R(a, c), R(c, a)) \geq i(h(u, v), h(z, t))$. It then suffices to prove that whenever $\min (x, y) \geq 0.6$, it holds that $i(h(u, v), h(z, t)) \geq 0.2$. Note that if $\min (x, y) \geq 0.6$, then also $\min (u, v, z, t) \geq 0.6$. We distinguish three cases:

(a) If $\max (u, v)=0.6$, then $u=v=0.6$. Since $i(u, z)=0.6$, it holds that $z>0.7$; analogously, $t>0.7$. Then $h(u, v)=T_{\mathbf{L}}(0.6,0.6)=0.2$ and $h(z, t)=T_{\mathbf{L}}(z, t)>0.4$, and $i(h(u, v), h(z, t))=0.2$.

(b) If $\max (z, t)=0.6$, then $z=t=0.6$. Since $i(u, z)=0.6$, it holds that $u>0.7$; analogously, $v>0.7$. Then $h(u, v)>0.4$ and as in (a) we obtain $i(h(u, v), h(z, t))=0.2$.

(c) If $\max (u, v)>0.6$ and $\max (z, t)>0.6$, then $h(u, v)>0.2$ and $h(z, t)>$ 0.2. Thus, $i(h(u, v), h(z, t)) \geq 0.2$.

Therefore, $f_{h}^{i}$ does not define the strongest transitivity that can be assured for I.

\subsection{The case of Frank t-norms}

An interesting problem is to know when the transitivity of $R$ is inherited by $I$, i.e. when departing from an $h$-transitive $R$, we can assure that $I$ is also $h$-transitive. We have answered this question in earlier work [19].

Theorem 4.2 Consider an increasing generator $i$ and a commutative conjunctor $h \leq T_{\mathbf{M}}$. Then the associated conjunctor $f_{h}^{i}$ equals $h$ if and only if $i$ dominates $h$.

This result applies in particular to $i=T_{\mathrm{M}}$ : an indifference relation $I$ generated from a reflexive fuzzy relation $R$ by means of the minimum operator, satisfies the same transitivity as $R$ does. Not only dominance allows us to obtain some general results, also the usual ordering of conjunctors, as is shown in the following corollary.

Corollary 4.3 For any bisymmetric increasing generator $i$ and any conjunctor $h$ such that $i \leq h \leq T_{\mathbf{M}}$, it holds that $f_{h}^{i}=i$. 
Proof. If $i$ is increasing and bisymmetric, then we have shown in [19] that the $h$-transitivity of $R$ is propagated into $i$-transitivity of $I$ when $i \leq h$. Theorem 4.1 then expresses that $i \leq f_{h}^{i}$. On the other hand, we have also proven in [19] that the transitivity of $I$ is upper bounded by $i$-transitivity, i.e. $f_{h}^{i} \leq i$. This concludes the proof.

Since every t-norm is increasing and bisymmetric, this result applies in particular to any generator that is a t-norm. In that case, transitivity of $R$ w.r.t. that t-norm is propagated to $I$. We will now invoke Theorem 4.2 and Corollary 4.3 to study the particular case of a Frank t-norm as generator.

Corollary 4.4 Consider $\lambda \in[0, \infty]$. It holds that $f_{T_{\mathbf{P}}}^{T_{\mathrm{M}}}=T_{\mathbf{P}}, f_{T_{\mathbf{L}}}^{T_{\lambda}^{\mathbf{F}}}=T_{\mathbf{L}}$ and $f_{h}^{T_{\lambda}^{\mathbf{F}}}=T_{\lambda}^{\mathbf{F}}$ for any $h \geq T_{\lambda}^{\mathbf{F}}$.

Proof. In the Frank t-norm family, only the following dominance relationships hold [36]:

$$
T_{0}^{\mathbf{F}}=T_{\mathbf{M}} \gg T_{\lambda}^{\mathbf{F}} \gg T_{\mathbf{L}}=T_{\infty}^{\mathbf{F}},
$$

for any $\lambda \in[0, \infty]$. Theorem 4.2 then yields $f_{T_{\mathbf{P}}}^{T_{\mathrm{M}}}=T_{\mathbf{P}}$ and $f_{T_{\mathbf{L}}}^{T_{\lambda}^{\mathrm{F}}}=T_{\mathbf{L}}$. On the other hand, Corollary 4.3 implies that $f_{h}^{T_{\lambda}^{\mathbf{F}}}=T_{\lambda}^{\mathbf{F}}$, provided that $T_{\lambda}^{\mathbf{F}} \leq h$.

Combining the results in Corollary 4.4 leads to Table 1. The entries in this table are the conjunctors $f_{h}^{i}$ that define the transitivity ensured for $I$ when the conjuntor $h$ that defines the transitivity of $R$ and the generator $i$ are one of the three most important t-norms.

$$
\begin{array}{c|ccc}
h \backslash i & T_{\mathbf{L}} & T_{\mathbf{P}} & T_{\mathbf{M}} \\
\hline T_{\mathbf{L}} & T_{\mathbf{L}} & T_{\mathbf{L}} & T_{\mathbf{L}} \\
T_{\mathbf{P}} & T_{\mathbf{L}} & T_{\mathbf{P}} & T_{\mathbf{P}} \\
T_{\mathbf{M}} & T_{\mathbf{L}} & T_{\mathbf{P}} & T_{\mathbf{M}}
\end{array}
$$

Table 1: Propagation of the transitivity of $R$ to $I$ for the three most important t-norms.

\section{Transitivity of strict preference relations}

\subsection{Main result}

This section is dedicated to strict preference relations. As shown in Subsection 2.2, given a generator $i$ and a large preference relation $R$, the strict preference relation $P$ is defined as $P=R-i\left(R, R^{t}\right)$. We study the minimal transitivity guaranteed for $P$ when we fix the transitivity of $R$ w.r.t. a conjunctor $h$. We start by recalling an upper bound for the transitivity of $P$ [19]. We know that the transitivity of $P$ is upper bounded by $h$-transitivity whenever $h \leq T_{\mathbf{M}}$. Next, we provide a full characterization of the transitivity of $P$. Compared to Theorem 4.1, we additionally require the 1-Lipschitz continuity of $i$. In other 
words, $i$ is considered to be a commutative quasi-copula. The commutativity of $h$ only serves the definition of $\mathcal{I}_{h}$. The additional condition on $h$ ensures that $\mathcal{I}_{h}$ is an implicator (see Proposition 3.1).

Theorem 5.1 Consider a 1-Lipschitz increasing generator $i$ and a commutative conjunctor $h$ such that $h(1, z)>0$ when $z>0$. For any reflexive fuzzy relation $R$ with corresponding strict preference relation $P$ generated by means of $i$, it holds that

$$
R \text { is h-transitive } \Rightarrow P \text { is } g_{h}^{i} \text {-transitive, }
$$

where $g_{h}^{i}$ is the conjunctor defined by

$g_{h}^{i}(x, y)=\inf _{\substack{1 \geq u \geq x \\ 1 \geq v \geq y}} h(u, v)-i\left(h(u, v), \min \left(\mathcal{I}_{h}\left(v, \mathcal{I}_{i}(u, u-x)\right), \mathcal{I}_{h}\left(u, \mathcal{I}_{i}(v, v-y)\right)\right)\right)$.

Moreover, this is the strongest result possible.

Proof. First, we prove that $g_{h}^{i}$ is a conjunctor. Since $i \leq T_{\mathbf{M}}$, it follows immediately that $g_{h}^{i}$ is a binary operation on $[0,1]$. Due to the additional condition on $h$, it holds that $\mathcal{I}_{h}$ is an implicator. Since $\mathcal{I}_{h}(1,0)=\mathcal{I}_{i}(1,0)=0$, it holds that

$$
g_{h}^{i}(1,1)=h(1,1)-i(h(1,1), \min (0,0))=1 .
$$

For $(x, y)=(1,0),(x, y)=(0,1)$ or $(x, y)=(0,0)$, it suffices to consider $(u, v)=$ $(x, y)$ to see that

$$
g_{h}^{i}(x, y) \leq h(x, y)-i\left(h(x, y), \min \left(\mathcal{I}_{h}\left(y, \mathcal{I}_{i}(x, 0)\right), \mathcal{I}_{h}\left(x, \mathcal{I}_{i}(y, 0)\right)\right)\right)=0,
$$

whence $g_{h}^{i}(1,0)=g_{h}^{i}(0,1)=g_{h}^{i}(0,0)=0$. On the other hand, $g_{h}^{i}$ has to be increasing. Let us introduce the following shorthand, for $(u, v) \geq(x, y)$,

$$
H_{x, y}(u, v)=h(u, v)-i\left(h(u, v), \min \left(\mathcal{I}_{h}\left(v, \mathcal{I}_{i}(u, u-x)\right), \mathcal{I}_{h}\left(u, \mathcal{I}_{i}(v, v-y)\right)\right)\right) .
$$

Let us consider $x_{1} \leq x_{2}$ and $y_{1} \leq y_{2}$. Since $\mathcal{I}_{h}$ and $\mathcal{I}_{i}$ are increasing in their second argument and $i$ is increasing, it holds for all $(u, v) \geq\left(x_{2}, y_{2}\right)$ that $H_{x_{2}, y_{2}}(u, v) \geq H_{x_{1}, y_{1}}(u, v)$, and thus

$$
\begin{aligned}
g_{h}^{i}\left(x_{2}, y_{2}\right) & =\inf _{u \geq x_{2}, v \geq y_{2}} H_{x_{2}, y_{2}}(u, v) \geq \inf _{u \geq x_{2}, v \geq y_{2}} H_{x_{1}, y_{1}}(u, v) \\
& \geq \inf _{u \geq x_{1}, v \geq y_{1}} H_{x_{1}, y_{1}}(u, v)=g_{h}^{i}\left(x_{1}, y_{1}\right) .
\end{aligned}
$$

Hence, $g_{h}^{i}$ is increasing and therefore is a conjunctor.

Second, we prove the implication. It suffices to prove that for any $(a, b, c) \in$ $A^{3}$ it holds that $g_{h}^{i}(P(a, b), P(b, c)) \leq P(a, c)$. Recall that since $i$ is 1-Lipschitz, it holds that for any fixed $k \in[0,1]$, the function $f(z)=z-i(z, k)$ is increasing. Let us call $u=R(a, b)$ and $v=R(b, c)$, then the $h$-transitivity of $R$ reads $R(a, c) \geq h(R(a, b), R(b, c))=h(u, v)$ and

$$
P(a, c)=R(a, c)-i(R(a, c), R(c, a)) \geq h(u, v)-i(h(u, v), R(c, a)) .
$$


The $h$-transitivity of $R$ also leads to $R(c, a) \leq \mathcal{I}_{h}(v, R(b, a))$ and $R(c, a) \leq$ $\mathcal{I}_{h}(u, R(c, b))$. Hence,

$$
R(c, a) \leq \min \left(\mathcal{I}_{h}(v, R(b, a)), \mathcal{I}_{h}(u, R(c, b))\right) .
$$

Let us call $x=P(a, b)$ and $y=P(b, c)$, then $x=u-i(u, R(b, a))$ is equivalent to $i(u, R(b, a))=u-x$ which implies $R(b, a) \leq \mathcal{I}_{i}(u, u-x)$. Similarly, $R(c, b) \leq$ $\mathcal{I}_{i}(v, v-y)$. Since $\mathcal{I}_{h}$ is increasing in its second argument, we obtain

$$
\begin{aligned}
R(c, a) & \leq \min \left(\mathcal{I}_{h}(v, R(b, a)), \mathcal{I}_{h}(u, R(c, b))\right) \\
& \leq \min \left(\mathcal{I}_{h}\left(v, \mathcal{I}_{i}(u, u-x)\right), \mathcal{I}_{h}\left(u, \mathcal{I}_{i}(v, v-y)\right)\right) .
\end{aligned}
$$

Combining the above leads to

$P(a, c) \geq \inf _{\substack{1 \geq u \geq x \\ 1 \geq v}} h(u, v)-i\left(h(u, v), \min \left(\mathcal{I}_{h}\left(v, \mathcal{I}_{i}(u, u-x)\right), \mathcal{I}_{h}\left(u, \mathcal{I}_{i}(v, v-y)\right)\right)\right)$,

i.e. $P(a, c) \geq g_{h}^{i}(P(a, b), P(b, c)$.

Finally, we prove that no greater conjunctor qualifies. Consider a conjunctor $g>g_{h}^{i}$, then we need to prove that there exists a reflexive fuzzy relation $R$ that is $h$-transitive, but for which the corresponding strict preference relation is not $g$-transitive. Since $g>g_{h}^{i}$, there exists $\left(x_{0}, y_{0}\right) \in[0,1]^{2}$ such that $g\left(x_{0}, y_{0}\right)>$ $g_{h}^{i}\left(x_{0}, y_{0}\right)$. Since

$$
g_{h}^{i}\left(x_{0}, y_{0}\right)=\inf _{\substack{1 \geq u \geq x_{0} \\ 1 \geq v \geq y_{0}}} H_{x_{0}, y_{0}}(u, v)
$$

and $\epsilon=g\left(x_{0}, y_{0}\right)-g_{h}^{i}\left(x_{0}, y_{0}\right)>0$, there exists $\left(u_{0}, v_{0}\right)$ such that $H_{x_{0}, y_{0}}\left(u_{0}, v_{0}\right)<$ $g_{h}^{i}\left(x_{0}, y_{0}\right)+\epsilon / 2$ and thus

$$
H_{x_{0}, y_{0}}\left(u_{0}, v_{0}\right)+\epsilon / 2<g(x, y) .
$$

Let us consider the reflexive fuzzy relation $R$ on $A=\{a, b, c\}$ given by

\begin{tabular}{c|ccc}
$R$ & $a$ & $b$ & $c$ \\
\hline$a$ & 1 & $u_{0}$ & $h\left(u_{0}, v_{0}\right)$ \\
$b$ & $\mathcal{I}_{i}\left(u_{0}, u_{0}-x_{0}\right)$ & 1 & $v_{0}$ \\
$c$ & $\min \left(z_{1}, z_{2}\right)$ & $\mathcal{I}_{i}\left(v_{0}, v_{0}-y_{0}\right)$ & 1
\end{tabular}

Here, $z_{1}=\max \left\{z \mid h\left(v_{0}, z\right) \leq \mathcal{I}_{i}\left(u_{0}, u_{0}-x_{0}\right)\right\}$ if this maximum exists. Otherwise, choose $\left.z_{1} \in\right] \mathcal{I}_{h}\left(v_{0}, \mathcal{I}_{i}\left(u_{0}, u_{0}-x_{0}\right)\right)-\epsilon / 2, \mathcal{I}_{h}\left(v_{0}, \mathcal{I}_{i}\left(u_{0}, u_{0}-x_{0}\right)\right)[$ such that $z_{1}>\mathcal{I}_{i}\left(u_{0}, u_{0}-x_{0}\right)$. In both cases, it holds that $h\left(v_{0}, z_{1}\right) \leq \mathcal{I}_{i}\left(u_{0}, u_{0}-x_{0}\right)$. Similarly, $z_{2}$ is chosen such that $h\left(u_{0}, z_{2}\right) \leq \mathcal{I}_{i}\left(v_{0}, v_{0}-y_{0}\right)$ and $z_{2}>\mathcal{I}_{i}\left(v_{0}, v_{0}-y_{0}\right)$. It then holds that $R$ is $h$-transitive.

On the other hand, it holds that

$$
\min \left(z_{1}, z_{2}\right)>\min \left(\mathcal{I}_{h}\left(v_{0}, \mathcal{I}_{i}\left(u_{0}, u_{0}-x_{0}\right)\right), \mathcal{I}_{h}\left(u_{0}, \mathcal{I}_{i}\left(v_{0}, v_{0}-y_{0}\right)\right)\right)-\epsilon / 2 .
$$

Since $i$ is 1-Lipschitz, it follows that

$$
\begin{aligned}
& i\left(h\left(u_{0}, v_{0}\right), \min \left(z_{1}, z_{2}\right)\right) \\
& \quad>i\left(h\left(u_{0}, v_{0}\right), \min \left(\mathcal{I}_{h}\left(v_{0}, \mathcal{I}_{i}\left(u_{0}, u_{0}-x_{0}\right)\right), \mathcal{I}_{h}\left(u_{0}, \mathcal{I}_{i}\left(v_{0}, v_{0}-y_{0}\right)\right)\right)\right)-\epsilon / 2
\end{aligned}
$$


and hence

$$
\begin{aligned}
& h\left(u_{0}, v_{0}\right)-i\left(h\left(u_{0}, v_{0}\right), \min \left(z_{1}, z_{2}\right)\right) \\
& <h\left(u_{0}, v_{0}\right)-i\left(h\left(u_{0}, v_{0}\right), \min \left(\mathcal{I}_{h}\left(u_{0}, \mathcal{I}_{i}\left(v_{0}, v_{0}-y\right)\right), \mathcal{I}_{h}\left(v_{0}, \mathcal{I}_{i}\left(u_{0}, u_{0}-x_{0}\right)\right)\right)\right)+\epsilon / 2 \\
& =H_{x_{0}, y_{0}}\left(u_{0}, v_{0}\right)+\epsilon / 2 .
\end{aligned}
$$

Consequently,

$$
\begin{aligned}
P(a, c) & =h\left(u_{0}, v_{0}\right)-i\left(h\left(u_{0}, v_{0}\right), \min \left(z_{1}, z_{2}\right)\right) \\
& <H_{x_{0}, y_{0}}\left(u_{0}, v_{0}\right)+\epsilon / 2<g\left(x_{0}, y_{0}\right)=g(P(a, b), P(b, c))
\end{aligned}
$$

and $P$ is not $g$-transitive.

One easily verifies that $g_{h}^{i}$ is indeed upper bounded by $h$ :

$g_{h}^{i}(x, y) \leq h(x, y)-i\left(h(x, y), \min \left(\mathcal{I}_{h}\left(v, \mathcal{I}_{i}(u, u-x)\right), \mathcal{I}_{h}\left(u, \mathcal{I}_{i}(v, v-y)\right)\right)\right) \leq h(x, y)$.

Remark 5.1 Note that the monotonicity of the generator $i$ is again necessary to ensure that the binary operation $g_{h}^{i}$ is a conjunctor. Indeed, consider the generator $i$ defined by

$$
i(x, y)=\left\{\begin{array}{l}
T_{\mathbf{L}}(x, y), \text { if } x+y>0.99 \\
T_{\mathbf{M}}(x, y), \text { otherwise }
\end{array}\right.
$$

and the conjunctor $h=T_{\mathbf{P}}$. In order to show that the operation $g_{h}^{i}$ is not increasing, it suffices to consider the points $(0.55,0.55)$ and $(0.6,0.6)$. Clearly, $g_{h}^{i}(0.6,0.6) \leq 0.6 \cdot 0.72-i\left(0.6 \cdot 0.72, \min \left(\mathcal{I}_{i}(0.6,0) / 0.72, \mathcal{I}_{i}(0.72,0.12) / 0.6\right)\right)=0$.

For $u, v \geq 0.55$, it holds that

$$
i\left(u v, \min \left(\mathcal{I}_{h}\left(v, \mathcal{I}_{i}(u, u-0.55)\right), \mathcal{I}_{h}\left(u, \mathcal{I}_{i}(v, v-0.55)\right)\right)\right)=i\left(u v, \frac{0.45}{\max (u, v)}\right) .
$$

One easily verifies that $u v+\frac{0.45}{\max (u, v)}>0.99$ for any $u, v \geq 0.55$, and hence

$$
i\left(u v, \frac{0.45}{\max (u, v)}\right)=\max \left(u v+\frac{0.45}{\max (u, v)}-1,0\right) .
$$

Hence,

$$
\begin{aligned}
& u v-i\left(u v, \min \left(\mathcal{I}_{h}\left(v, \mathcal{I}_{i}(u, u-0.55)\right), \mathcal{I}_{h}\left(u, \mathcal{I}_{i}(v, v-0.55)\right)\right)\right) \\
& =u v-\max \left(u v+\frac{0.45}{\max (u, v)}-1,0\right) \\
& = \begin{cases}1-\frac{0.45}{\max (u, v)} \geq 1-\frac{0.45}{0.55}=0.1818 & , \text { if } u v+\frac{0.45}{\max (u, v)}>1, \\
u v \geq 0.55^{2}=0.3025 & \text {, otherwise. }\end{cases}
\end{aligned}
$$

Consequently, $g_{h}^{i}(0.55,0.55) \geq 0.1818>g_{h}^{i}(0.6,0.6)=0$. 


\subsection{The case of 1-Lipschitz t-norms}

In the particular case when the conjunctor $h$, expressing the $h$-transitivity of $R$, and the indifference generator $i$ are one and the same t-norm, the thorny general expression obtained in Theorem 5.1 gets much simpler. Compared to Theorem 5.1, we additionally require the associativity of $i$ and consider $h$ to be identical to $i$.

Note that for a left-continuous t-norm $T$, it holds that $\mathcal{I}_{T}(T(x, y), z)=$ $\mathcal{I}_{T}\left(x, \mathcal{I}_{T}(y, z)\right)$, for any $(x, y, z) \in[0,1]^{3}$. Also, for a continuous t-norm $T$, it holds that $T\left(x, \mathcal{I}_{T}(x, y)\right)=\min (x, y)$, for any $(x, y) \in[0,1]^{2}$ (see also Lemma 3.2). See e.g. [25] for more details. These properties will be used in the proof of the following theorem.

Theorem 5.2 Consider a 1-Lipschitz t-norm T. For any reflexive fuzzy relation $R$ with corresponding strict preference relation $P$ generated by means of $T$, it holds that

$$
R \text { is } T \text {-transitive } \Rightarrow P \text { is } g_{T}^{T} \text {-transitive, }
$$

where $g_{T}^{T}$ is the conjunctor defined by

$$
g_{T}^{T}(x, y)=\inf _{0 \leq \alpha \leq \min (1-x, 1-y)} \max (T(x+\alpha, y+\alpha)-\alpha, 0) .
$$

Moreover, this is the strongest result possible.

Proof. First of all, we will prove that

$$
g_{T}^{T}(x, y)=\inf _{\substack{1 \geq u \geq x \\ 1 \geq v \geq y}} \max (T(u, v)-\min (u-x, v-y), 0) .
$$

To obtain this expression from the general expression in Theorem 5.1, it suffices to prove that

$T\left(T(u, v), \min \left(\mathcal{I}_{T}\left(v, \mathcal{I}_{T}(u, u-x)\right), \mathcal{I}_{T}\left(u, \mathcal{I}_{T}(v, v-y)\right)\right)\right)=\min (u-x, v-y, T(u, v))$.

Let $z_{1}=\mathcal{I}_{T}\left(v, \mathcal{I}_{T}(u, u-x)\right)$ and $z_{2}=\mathcal{I}_{T}\left(u, \mathcal{I}_{T}(v, v-y)\right)$, then it holds that $z_{1}=\mathcal{I}_{T}(T(u, v), u-x)$ and $z_{2}=\mathcal{I}_{T}(T(u, v), v-y)$. Hence, $\min \left(z_{1}, z_{2}\right)=$ $\mathcal{I}_{T}(T(u, v), \min (u-x, v-y))$ and

$$
\begin{aligned}
& T\left(T(u, v), \min \left(\mathcal{I}_{T}\left(v, \mathcal{I}_{T}(u, u-x)\right), \mathcal{I}_{T}\left(u, \mathcal{I}_{T}(v, v-y)\right)\right)\right) \\
= & T\left(T(u, v), \mathcal{I}_{T}(T(u, v), \min (u-x, v-y))\right)=\min (u-x, v-y, T(u, v)) .
\end{aligned}
$$

The conjunctor $g_{T}^{T}$ can then be written as

$$
g_{T}^{T}(x, y)=\inf _{\substack{1 \geq u \geq x \\ 1 \geq v \geq y}} \max (T(u, v)-\min (u-x, v-y), 0) .
$$

If we now replace $u$ and $v$ by $x+\alpha$ and $y+\beta$, with $\alpha \in[0,1-x]$ and $\beta \in[0,1-y]$, we obtain

$$
g_{T}^{T}(x, y)=\inf _{\substack{0 \leq \alpha \leq 1-x \\ 0 \leq \beta \leq 1-y}} \max (T(x+\alpha, y+\beta)-\min (\alpha, \beta), 0) .
$$


If $\alpha \leq \beta$, then it holds that

$$
\begin{aligned}
\max (T(x+\alpha, y+\beta)-\min (\alpha, \beta), 0) & =\max (T(x+\alpha, y+\beta)-\alpha, 0) \\
& \geq \max (T(x+\alpha, y+\alpha)-\alpha, 0) .
\end{aligned}
$$

Similarly, if $\beta \leq \alpha$, it holds that

$$
\max (T(x+\alpha, y+\beta)-\min (\alpha, \beta), 0) \geq \max (T(x+\beta, y+\beta)-\beta, 0) .
$$

Thus,

$$
\begin{aligned}
& \inf _{\substack{0 \leq \alpha \leq 1-x \\
0 \leq \beta \leq 1-y}} \max (T(x+\alpha, y+\beta)-\min (\alpha, \beta), 0) \\
& \geq \inf _{0 \leq \alpha \leq \min (1-x, 1-y)} \max (T(x+\alpha, y+\alpha)-\alpha, 0) .
\end{aligned}
$$

As the opposite inequality holds trivially, this ends the proof. $\square$

\subsection{The case of Frank t-norms}

The above theorem addresses 1-Lipschitz t-norms. Such operations can be equivalently described as associative copulas [30]. In [8] the conjunctor $g_{T}^{T}$ was studied in depth not only for a t-norm $T$, but for a general binary aggregation function. In particular, for the Frank t-norms/copulas, the expression for $g_{T}^{T}$ can be further simplified. Consider $\lambda \in[0, \infty]$, then it holds that [8]:

$$
g_{T_{\lambda}^{\mathrm{F}}}^{T_{\lambda}^{\mathbf{F}}}= \begin{cases}T_{\lambda}^{\mathbf{F}}(x, y) & , \text { if } x+y>1, \\ \max \left(T_{\lambda}^{\mathbf{F}}\left(\frac{1+x-y}{2}, \frac{1+y-x}{2}\right)-\frac{1-x-y}{2}, 0\right), \text { otherwise. }\end{cases}
$$

In particular, it holds that $g_{T_{\mathrm{L}}}^{T_{\mathrm{L}}}=T_{\mathbf{L}}, g_{T_{\mathrm{M}}}^{T_{\mathrm{M}}}=T_{\mathrm{M}}$ (directly obtained in [16]) and

$$
g_{T_{\mathbf{P}}}^{T_{\mathbf{P}}}(x, y)=\left(T_{\mathbf{P}}(x, y)-\left(\frac{T_{\mathbf{L}}(1-x, 1-y)}{2}\right)^{2}\right) \cdot \chi_{] 1, \infty[}(\sqrt{x}+\sqrt{y}) .
$$

Remark 5.2 In [8], a non-trivial proof shows that for any Frank $t$-norm/copula $T_{\lambda}^{\mathbf{F}}$, the conjunctor $g_{T_{\lambda}^{\mathbf{F}}}^{T^{\mathbf{F}}}$ is a copula as well. However, for $\left.\lambda \in\right] 0, \infty[$, the conjunctor $g_{T_{\lambda}^{\mathrm{F}}}^{T_{\lambda}^{\mathrm{F}}}$ is not a t-norm. Let us show that it is not associative. Consider $x, z \in] 0,1[$ such that $x>1-z>0.5$. It holds that

$$
\phi_{\lambda}^{-1}\left(\frac{\phi_{\lambda}(1-z)}{\phi_{\lambda}(x)}\right)>1-z .
$$

On the other hand, the function $f(t)=\phi_{\lambda}(t) \phi_{\lambda}(1-t)$ is strictly increasing on $[0,0.5]$ and strictly decreasing on $[0.5,1]$, whence $\phi_{\lambda}(x) \phi_{\lambda}(1-x)<\phi_{\lambda}(z) \phi_{\lambda}(1-$ $z)$, which implies that

$$
\phi_{\lambda}^{-1}\left(\frac{\phi_{\lambda}(1-x)}{\phi_{\lambda}(z)}\right)<\phi_{\lambda}^{-1}\left(\frac{\phi_{\lambda}(1-z)}{\phi_{\lambda}(x)}\right) .
$$


Thus, for any $\lambda \in] 0, \infty[$, the interval

$$
] \max \left(1-z, \phi_{\lambda}^{-1}\left(\frac{\phi_{\lambda}(1-x)}{\phi_{\lambda}(z)}\right)\right), \phi_{\lambda}^{-1}\left(\frac{\phi_{\lambda}(1-z)}{\phi_{\lambda}(x)}\right)[
$$

is not empty.

Let us now consider a value $y$ in this interval. It then holds that $x+y>1$, $y+z>1, T_{\lambda}^{\mathbf{F}}(x, y)<1-z$ and $T_{\lambda}^{\mathbf{F}}(y, z)>1-x$. The triplet $(x, y, z)$ then satisfies

$$
\begin{aligned}
g_{T_{\lambda}^{\mathrm{F}}}^{T_{\mathrm{F}}^{\mathrm{F}}}\left(g_{T_{\lambda}^{\mathrm{F}}}^{T_{\mathrm{F}}^{\mathrm{F}}}(x, y), z\right) & =g_{T_{\lambda}^{\mathrm{F}}}^{T^{\mathbf{F}}}\left(T_{\lambda}^{\mathbf{F}}(x, y), z\right) \\
& <T_{\lambda}^{\mathbf{F}}\left(T_{\lambda}^{\mathbf{F}}(x, y), z\right)=T_{\lambda}^{\mathbf{F}}\left(x, T_{\lambda}^{\mathbf{F}}(y, z)\right)=g_{T_{\lambda}^{T_{\lambda}^{\mathrm{F}}}}^{T^{\mathrm{F}}}\left(x, g_{T_{\lambda}^{\mathrm{F}}}^{T^{\mathbf{F}}}(y, z)\right),
\end{aligned}
$$

where the inequality comes from the fact that $g_{T_{\lambda}^{\mathrm{F}}}^{T_{\lambda}^{\mathrm{F}}}(u, v)<T_{\lambda}^{\mathbf{F}}(u, v)$ whenever $u+v<1$. Hence, $g_{T_{\lambda}^{\mathrm{F}}}^{T_{\mathrm{F}}^{\mathrm{F}}}$ is not associative and therefore it is not a t-norm. In particular, $g_{T_{\mathbf{P}}}^{T_{\mathrm{P}}}$ is not a t-norm. This seems to indicate that in the present context, the 2-increasingness property plays a more prominent role than the associativity property.

Next, we prove a lemma that will turn out useful in the following proposition.

Lemma 5.3 For any $\lambda \in] 0, \infty\left[\right.$ and any $(x, y) \in[0,1]^{2}$ such that $x+y>1$, the function $H_{x, y}:[x, 1] \times[y, 1] \rightarrow[0,1]$ defined by $H_{x, y}(u, v)=$

$$
\phi_{\lambda}^{-1}\left(\phi_{\lambda}(u+v-1) \phi_{\lambda}\left(\max \left(u+\phi_{\lambda}^{-1}\left(\frac{\phi_{\lambda}(y)}{\phi_{\lambda}(v)}\right), v+\phi_{\lambda}^{-1}\left(\frac{\phi_{\lambda}(x)}{\phi_{\lambda}(u)}\right)\right)-1\right)\right)
$$

reaches its minimum at $(u, v)=(x, y)$.

Proof. Since the function $\phi_{\lambda}^{-1}$ is strictly increasing for any $\left.\lambda \in\right] 0, \infty[$, we have to show that the function $h_{x, y}:[x, 1] \times[y, 1] \rightarrow[0,1]$ defined by

$h_{x, y}(u, v)=\phi_{\lambda}(u+v-1) \phi_{\lambda}\left(\max \left(u+\phi_{\lambda}^{-1}\left(\frac{\phi_{\lambda}(y)}{\phi_{\lambda}(v)}\right)-1, v+\phi_{\lambda}^{-1}\left(\frac{\phi_{\lambda}(x)}{\phi_{\lambda}(u)}\right)-1\right)\right)$

reaches its minimum at $(u, v)=(x, y)$. Let us define

$$
\begin{aligned}
& h_{x, y}^{1}(u, v)=\phi_{\lambda}(u+v-1) \phi_{\lambda}\left(u+\phi_{\lambda}^{-1}\left(\frac{\phi_{\lambda}(y)}{\phi_{\lambda}(v)}\right)-1\right) \\
& h_{x, y}^{2}(u, v)=\phi_{\lambda}(u+v-1) \phi_{\lambda}\left(\phi_{\lambda}^{-1}\left(\frac{\phi_{\lambda}(x)}{\phi_{\lambda}(u)}\right)+v-1\right) .
\end{aligned}
$$

Since $\phi_{\lambda}$ is increasing, it holds that $h_{x, y}(u, v)=\max \left(h_{x, y}^{1}(u, v), h_{x, y}^{2}(u, v)\right)$ and

$$
\min _{\substack{1 \geq v \geq y \\ 1 \geq u \geq x}} h_{x, y}(u, v) \geq \max \left(\min _{\substack{1 \geq v \geq y \\ 1 \geq u \geq x}} h_{x, y}^{1}(u, v), \min _{\substack{1 \geq v \geq y \\ 1 \geq u \geq x}} h_{x, y}^{2}(u, v)\right) \text {. }
$$


The function $h_{x, y}^{1}$ is increasing in its first argument, whence

$$
\min _{\substack{1 \geq v \geq y \\ 1 \geq u \geq x}} h_{x, y}^{1}(u, v)=\min _{1 \geq v \geq y} \phi_{\lambda}(x+v-1) \phi_{\lambda}\left(x+\phi_{\lambda}^{-1}\left(\frac{\phi_{\lambda}(y)}{\phi_{\lambda}(v)}\right)-1\right) .
$$

Similarly, $h_{x, y}^{2}$ is increasing in its second argument, whence

$$
\min _{\substack{1 \geq v \geq y \\ 1 \geq u \geq x}} h_{x, y}^{2}(u, v)=\min _{1 \geq u \geq x} \phi_{\lambda}(u+y-1) \phi_{\lambda}\left(y+\phi_{\lambda}^{-1}\left(\frac{\phi_{\lambda}(x)}{\phi_{\lambda}(u)}\right)-1\right) .
$$

These two right-hand sides determine functions of the same type:

- For $\lambda=1$, these functions are of the form

$$
F_{1}(z)=(z-k)\left(\frac{c}{z}-k\right)
$$

where $k \in[0,1[, c \in] 0,1]$ and $z \in[c, 1]$. Its derivative is given by $F_{1}^{\prime}(z)=$ $-k+\frac{k c}{z^{2}}$, whence $F_{1}(z)$ is increasing on $[c, \sqrt{c}$ and decreasing on $[\sqrt{c}, 1]$. Hence,

$$
\min _{z \in[c, 1]} F_{1}(z) \in\left\{F_{1}(c), F_{1}(1)\right\} .
$$

- For $\lambda>1$, these functions are of the form

$$
F_{\lambda}(z)=K\left(k \lambda^{z}-1\right)\left(k\left(1+\frac{\left(\lambda^{c}-1\right)(\lambda-1)}{\left(\lambda^{z}-1\right)}\right)-1\right),
$$

where $K>0, k \in] 0,1], c \in] 0,1]$ and $z \in[c, 1]$. Let us denote $C=$ $\left(\lambda^{c}-1\right)(\lambda-1)$, then the function $F_{\lambda}(z)$ can be written as

$$
\begin{aligned}
F_{\lambda}(z) & =K\left(k \lambda^{z}-1\right)\left(k\left(1+\frac{C}{\lambda^{z}-1}\right)-1\right) \\
& =K\left(k^{2} \lambda^{z}+\frac{C k^{2} \lambda^{z}}{\lambda^{z}-1}-k \lambda^{z}-k-\frac{C k}{\lambda^{z}-1}+1\right),
\end{aligned}
$$

while its derivative is given by

$$
\begin{aligned}
F_{\lambda}^{\prime}(z) & =K k \lambda^{z} \ln (\lambda)\left[k-\frac{C k}{\left(\lambda^{z}-1\right)^{2}}-1+\frac{C}{\left(\lambda^{z}-1\right)^{2}}\right] \\
& =K k \lambda^{z} \ln (\lambda)(1-k)\left(\frac{C}{\left(\lambda^{z}-1\right)^{2}}-1\right) .
\end{aligned}
$$

If $k=1$, the function $F_{\lambda}$ is constant. Otherwise, the following equivalences hold:

$$
\begin{array}{lcccc}
F_{\lambda}^{\prime}(z)>0 & \text { iff } & \frac{C}{\left(\lambda^{z}-1\right)^{2}}>1 & \text { iff } & z<\log _{\lambda}\left(1+\sqrt{\left(\lambda^{c}-1\right)(\lambda-1)}\right) \\
F_{\lambda}^{\prime}(z)<0 & \text { iff } & \frac{C}{\left(\lambda^{z}-1\right)^{2}}<1 & \text { iff } & z>\log _{\lambda}\left(1+\sqrt{\left(\lambda^{c}-1\right)(\lambda-1)}\right) .
\end{array}
$$


Since $0<c \leq \log _{\lambda}\left(1+\sqrt{\left(\lambda^{c}-1\right)(\lambda-1)}\right) \leq 1$, the function $F_{\lambda}(z)$ is increasing on $\left[c, \log _{\lambda}\left(1+\sqrt{\left(\lambda^{c}-1\right)(\lambda-1)}\right)\right]$ and decreasing on $\left[\log _{\lambda}(1+\right.$ $\left.\left.\sqrt{\left(\lambda^{c}-1\right)(\lambda-1)}\right), 1\right]$. It therefore reaches its minimum at $z=c$ or $z=1$.

- For $\lambda<1$, these functions are of the form

$$
F_{\lambda}(z)=K\left(1-k \lambda^{z}\right)\left(1-k\left(1-\frac{C}{\left(1-\lambda^{z}\right)}\right)\right),
$$

where $K>0, k \geq 1, c \in] 0,1]$ and $z \in[c, 1]$, and $C=\left(1-\lambda^{c}\right)(1-\lambda)$. Its derivative is given by

$$
F_{\lambda}^{\prime}(z)=K k \lambda^{z} \ln (\lambda)(1-k)\left(\frac{C}{\left(1-\lambda^{z}\right)^{2}}-1\right)
$$

where $\ln (\lambda)<0$ since $\lambda \in] 0,1\left[\right.$. Hence, if $k=1$, the function $F_{\lambda}$ is constant, while otherwise it is increasing on $\left[c, \log _{\lambda}\left(1-\sqrt{\left(1-\lambda^{c}\right)(1-\lambda)}\right)\right]$ and decreasing on $\left[\log _{\lambda}\left(1-\sqrt{\left(1-\lambda^{c}\right)(1-\lambda)}\right), 1\right]$. Also in this case, the minimum is reached at $z=c$ or $z=1$.

Since $h_{x, y}^{1}(x, y)=\phi_{\lambda}(x+y-1) \phi_{\lambda}(x)=h_{x, y}^{1}(x, 1)$ and similarly $h_{x, y}^{2}(x, y)=$ $h_{x, y}^{2}(1, y)$, we can conclude that

$$
\max \left(\min _{\substack{1 \geq v \geq y \\ 1 \geq u \geq x}} h_{x, y}^{1}(u, v), \min _{\substack{1 \geq v \geq y \\ 1 \geq u \geq x}} h_{x, y}^{2}(u, v)\right)=\max \left(h_{x, y}^{1}(x, y), h_{x, y}^{2}(x, y)\right) .
$$

However, this value is reached by $h_{x, y}(u, v)$ at $(u, v)=(x, y)$, and thus

$$
\min _{\substack{1 \geq v \geq y \\ 1 \geq u \geq x}} h_{x, y}(u, v)=h_{x, y}(x, y)
$$

which completes the proof. $\square$

Other particular cases involving the three most important t-norms, i.e. both $i$ and $h$ belonging to $\left\{T_{\mathbf{L}}, T_{\mathbf{P}}, T_{\mathbf{M}}\right\}$, are instances of the following propositions.

Proposition 5.4 Consider $\lambda \in[0, \infty]$. For $i=T_{\lambda}^{\mathbf{F}}$ and $h=T_{\mathbf{L}}$, it holds that

$$
g_{T_{\mathbf{L}}}^{T_{\lambda}^{\mathbf{F}}}(x, y)=T_{1 / \lambda}^{\mathbf{F}}\left(T_{\mathbf{L}}(x, y), S_{\mathbf{M}}(x, y)\right) .
$$

Proof. We have already indicated that $g_{T_{\mathbf{L}}}^{T_{\mathbf{L}}}(x, y)=T_{\mathbf{L}}(x, y)=T_{\mathbf{M}}\left(T_{\mathbf{L}}(x, y), S_{\mathbf{M}}(x, y)\right)$. It is also easy to prove that $g_{T_{\mathbf{L}}}^{T_{\mathrm{M}}}(x, y)=T_{\mathbf{L}}\left(T_{\mathbf{L}}(x, y), S_{\mathbf{M}}(x, y)\right)$. To prove the general expression for $\lambda \in] 0, \infty[$, we notice first of all that from the equality

$$
T_{1 / \lambda}^{\mathbf{F}}(x, y)=x-T_{\lambda}^{\mathbf{F}}(x, 1-y)
$$

it follows that

$$
\mathcal{I}_{T_{\lambda}^{\mathrm{F}}}(x, y)=1-\mathcal{I}_{T_{1 / \lambda}^{\mathrm{F}}}(x, x-y)
$$


Now, from Theorem 5.1 we obtain

$$
\begin{aligned}
& g_{T_{\mathbf{L}}}^{T_{\mathbf{\mathbf { T }}}^{\mathbf{F}}}(x, y) \\
& =\inf _{\substack{1 \geq u \geq x \\
1 \geq v \geq y}} T_{\mathbf{L}}(u, v)-T_{\lambda}^{\mathbf{F}}\left(T_{\mathbf{L}}(u, v), \min \left(1+\mathcal{I}_{T_{\lambda}^{\mathbf{F}}}(u, u-x)-v, 1+\mathcal{I}_{T_{\lambda}^{\mathbf{F}}}(v, v-y)-u\right)\right) \\
& =\inf _{\substack{1 \geq u \geq x \\
1 \geq v \geq y}} T_{1 / \lambda}^{\mathbf{F}}\left(T_{\mathbf{L}}(u, v), \max \left(v+\mathcal{I}_{T_{1 / \lambda}^{\mathbf{F}}}(u, x)-1, u+\mathcal{I}_{T_{1 / \lambda}^{\mathbf{F}}}(v, y)-1\right)\right) .
\end{aligned}
$$

We proved in Lemma 5.3 that this expression reaches its minimum at $(u, v)=$ $(x, y)$

Proposition 5.5 Consider $\lambda \in[0, \infty]$. For $i=T_{\lambda}^{\mathbf{F}}$ and $h=T_{\mathbf{M}}$, it holds that

$$
g_{T_{\mathbf{M}}}^{T_{\lambda}^{\mathbf{F}}}(x, y)=T_{\mathbf{n M}}^{\varphi_{1 / \lambda}}(x, y)= \begin{cases}\min (x, y) & , \text { if } \varphi_{1 / \lambda}(x)+\varphi_{1 / \lambda}(y)>1 \\ 0 & , \text { otherwise }\end{cases}
$$

where $\varphi_{\lambda}$ is defined by

$$
\varphi_{\lambda}(x)= \begin{cases}\log _{\lambda}\left(\sqrt{\frac{\lambda^{x}-1}{\lambda-1}}(\lambda-1)+1\right) & , \text { if } \lambda \in] 0,1[\cup] 1, \infty[, \\ x & , \text { if } \lambda=0 \\ \sqrt{x} & \text {, if } \lambda=1 \\ \frac{x+1}{2} \chi_{] 0,1]}(x) & , \text { if } \lambda=\infty\end{cases}
$$

Moreover, $g_{T_{\mathbf{M}}}^{T_{\lambda}^{\mathbf{F}}}$ is a t-norm.

Proof. The expression for $g_{T_{\mathbf{M}}}^{T_{\lambda}^{\mathbf{F}}}$ was obtained in [16]. Clearly, $g_{T_{\mathbf{M}}}^{T_{\mathbf{L}}}=T_{\mathbf{n M}}$ and $g_{T_{\mathrm{M}}}^{T_{\mathrm{M}}}=T_{\mathbf{M}}$. As $\varphi_{\lambda}$ is an automorphism for any $\left.\lambda \in\right] 0, \infty\left[, g_{T_{\mathbf{M}}}^{T_{\mathbf{Y}}^{\mathbf{F}}}\right.$ is a $\phi$-transform of $T_{\mathbf{n M}}$ and is therefore also a t-norm. $\square$

As a consequence of this proposition, we obtain that $g_{T_{\mathbf{M}}}^{T_{\mathbf{L}}}=T_{\mathbf{n M}}$ and

$$
g_{T_{\mathbf{M}}}^{T_{\mathbf{P}}}(x, y)=T_{\mathbf{n M}}^{\varphi_{1}}(x, y)= \begin{cases}\min (x, y) & , \text { if } \sqrt{x}+\sqrt{y}>1 \\ 0 & , \text { otherwise }\end{cases}
$$

In order to complete the study of all combinations of the three most important t-norms, two cases are missing, namely $g_{T_{\mathbf{P}}}^{T_{\mathbf{L}}}$ and $g_{T_{\mathbf{P}}}^{T_{\mathrm{M}}}$. These will be addressed in Propositions 5.6 and 5.7.

Proposition 5.6 For $i=T_{\mathbf{L}}$ and $h=T_{\mathbf{P}}$, it holds that

$$
g_{T_{\mathbf{P}}}^{T_{\mathbf{L}}}(x, y)=T_{\mathbf{M}}\left(T_{\mathbf{P}}(x, y), \frac{T_{\mathbf{L}}(x, y)}{T_{\mathbf{M}}(x, y)}\right) \cdot \chi_{] 0,1]}\left(T_{\mathbf{M}}(x, y)\right) .
$$


Proof. If $x+y \leq 1$, then $g_{T_{\mathbf{P}}}^{T_{\mathbf{L}}}(x, y) \leq g_{T_{\mathbf{P}}}^{T_{\mathbf{L}}}(x, 1-x)=0$. Also $T_{\mathbf{M}}\left(T_{\mathbf{P}}(x, y), \frac{T_{\mathbf{L}}(x, y)}{T_{\mathbf{M}}(x, y)}\right)=$ 0 . Thus, in this case, both expressions are equal. Next, consider $x+y>1$. From Theorem 5.1 we obtain

$$
\begin{aligned}
g_{T_{\mathbf{P}}}^{T_{\mathbf{L}}}(x, y) & =\inf _{\substack{1 \geq u \geq x \\
1 \geq v \geq y}}\left(u v-\max \left(u v+\min \left(\frac{1-x}{v}, \frac{1-y}{u}\right)-1,0\right)\right) \\
& =\inf _{\substack{1 \geq u \geq x \\
1 \geq v \geq y}} \min \left(1-\min \left(\frac{1-x}{v}, \frac{1-y}{u}\right), u v\right) \\
& =\inf _{\substack{1 \geq u \geq x \\
1 \geq v \geq y}} \min \left(\max \left(\frac{v+x-1}{v}, \frac{u+y-1}{u}\right), u v\right) .
\end{aligned}
$$

For $(u, v)=(x, y)$, the right-hand side yields $T_{\mathbf{M}}\left(T_{\mathbf{P}}(x, y), \frac{T_{\mathbf{L}}(x, y)}{T_{\mathbf{M}}(x, y)}\right)$ and therefore $g_{T_{\mathbf{P}}}^{T_{\mathbf{L}}}(x, y) \leq T_{\mathbf{M}}\left(T_{\mathbf{P}}(x, y), \frac{T_{\mathbf{L}}(x, y)}{T_{\mathbf{M}}(x, y)}\right)$. It then suffices to prove that for any $(u, v) \geq(x, y)$ it holds that

$$
\min \left(\max \left(\frac{v+x-1}{v}, \frac{u+y-1}{u}\right), u v\right) \geq T_{\mathbf{M}}\left(T_{\mathbf{P}}(x, y), \frac{T_{\mathbf{L}}(x, y)}{T_{\mathbf{M}}(x, y)}\right) .
$$

On the one hand, $u v \geq T_{\mathbf{P}}(x, y) \geq T_{\mathbf{M}}\left(T_{\mathbf{P}}(x, y), \frac{T_{\mathbf{L}}(x, y)}{T_{\mathbf{M}}(x, y)}\right)$. On the other hand,

$$
\max \left(\frac{v+x-1}{v}, \frac{u+y-1}{u}\right)=\max \left(1-\frac{1-x}{v}, 1-\frac{1-y}{u}\right) .
$$

Let us assume w.l.o.g. that $y \leq x$. Since $v \geq y$, it holds that

$$
1-\frac{1-x}{v} \geq 1-\frac{1-x}{y}=\frac{T_{\mathbf{L}}(x, y)}{y} \geq \frac{T_{\mathbf{L}}(x, y)}{T_{\mathbf{M}}(x, y)} .
$$

This concludes the proof. $\square$

Remark 5.3 The conjunctor $g_{T_{\mathbf{P}}}^{T_{\mathbf{L}}}$ is not a t-norm. Associativity is violated, as, for instance,

$$
g_{T_{\mathbf{P}}}^{T_{\mathbf{L}}}\left(g_{T_{\mathbf{P}}}^{T_{\mathbf{L}}}(0.5,0.9), 0.6\right)=\frac{1}{9} \neq \frac{2}{25}=g_{T_{\mathbf{P}}}^{T_{\mathbf{L}}}\left(0.5, g_{T_{\mathbf{P}}}^{T_{\mathbf{L}}}(0.9,0.6)\right) .
$$

Proposition 5.7 For $i=T_{\mathbf{M}}$ and $h=T_{\mathbf{P}}$, it holds that

$$
g_{T_{\mathbf{P}}}^{T_{\mathrm{M}}}(x, y)=\max \left(\min _{\substack{1 \geq u \geq x \\ 1 \geq v \geq y}}\left(u v-\min \left(\frac{u-x}{v}, \frac{v-y}{u}\right)\right), 0\right) \cdot \chi_{[0,1]^{2}}(x, y) .
$$


The proof of this equality left for last, on purpose, is the most cumbersome one, as it does not lead to a closed analytical expression. Indeed, tedious calculations (of which we spare the reader) lead to

$g_{T_{\mathbf{P}}}^{T_{\mathbf{M}}}(x, y)= \begin{cases}0 & , \text { if } G_{\mathbf{P}}^{\mathbf{M}}(x, y) \leq 0, \\ g\left(\alpha_{x, y}, \frac{y+\sqrt{y^{2}+4 \alpha_{x, y}\left(\alpha_{x, y}-x\right)}}{2}, x, y\right) & , \text { if } G_{\mathbf{P}}^{\mathbf{M}}(x, y)>0 \text { and } \exists \alpha_{x, y}, \\ T_{\mathbf{P}}(x, y) & , \text { if } G_{\mathbf{P}}^{\mathbf{M}}(x, y)>0 \text { and } \exists \alpha_{x, y},\end{cases}$

where

$$
\begin{gathered}
g(u, v, x, y)=T_{\mathbf{P}}(u, v)-T_{\mathbf{M}}\left(\frac{u-x}{v}, \frac{v-y}{u}\right) \\
G_{\mathbf{P}}^{\mathbf{M}}(x, y)=256\left(x^{2}+y^{2}-1\right)^{3}+27 x^{2} y^{2}\left(x^{2} y^{2}-32 x^{2}-32 y^{2}+160\right)
\end{gathered}
$$

and $\alpha_{x, y}$ is a solution in $[x, 1]$ of the following equation in the variable $u$ :

$$
\left(4 u^{3}-3 x u^{2}-x\right)^{2}=y^{2}\left(-3 u^{4}+2 x u^{3}-6 u^{2}+6 x u+1-y^{2}\right) .
$$

Note that neither the existence nor the uniqueness of such a solution is guaranteed. However, in case there exists more than one solution, all of them lead to the same value of $g\left(\alpha_{x, y}, \frac{y+\sqrt{y^{2}+4 \alpha_{x, y}\left(\alpha_{x, y}-x\right)}}{2}, x, y\right)$, which guarantees that $g_{T_{\mathrm{P}}}^{T_{\mathrm{M}}}$ is properly defined.

In this subsection we have obtained, among many other results, that

$$
\begin{aligned}
& g_{T_{\mathbf{P}}}^{T_{\mathbf{L}}}(x, y)=T_{\mathbf{M}}\left(T_{\mathbf{P}}(x, y), \frac{T_{\mathbf{L}}(x, y)}{T_{\mathbf{M}}(x, y)}\right) \cdot \chi_{] 0,1]}\left(T_{\mathbf{M}}(x, y)\right), \\
& g_{T_{\mathbf{P}}}^{T_{\mathbf{P}}}(x, y)=\left(T_{\mathbf{P}}(x, y)-\left(\frac{T_{\mathbf{L}}(1-x, 1-y)}{2}\right)^{2}\right) \cdot \chi_{] 1, \infty[}(\sqrt{x}+\sqrt{y}), \\
& g_{T_{\mathbf{P}}}^{T_{\mathbf{M}}}(x, y)= \begin{cases}0 & , \text { if } G_{\mathbf{P}}^{\mathbf{M}}(x, y) \leq 0, \\
g\left(\alpha_{x, y}, \frac{y+\sqrt{y^{2}+4 \alpha_{x, y}\left(\alpha_{x, y}-x\right)}}{2}, x, y\right), & \text { if } G_{\mathbf{P}}^{\mathbf{M}}(x, y)>0 \text { and } \exists \alpha_{x, y}, \\
T_{\mathbf{P}}(x, y) & \text { if } G_{\mathbf{P}}^{\mathbf{M}}(x, y)>0 \text { and } \nexists \alpha_{x, y} .\end{cases}
\end{aligned}
$$

Combining the results in Propositions 5.4-5.7 and the previous expressions leads to Table 2. The entries in this table are the conjunctors $g_{h}^{i}$ that define the transitivity ensured for $P$ when the conjuntor $h$ that defines the transitivity of $R$ and the generator $i$ are one of the three most important t-norms.

\section{Conclusion}

We have dealt with the transitivity of indifference and strict preference relations generated by means of a generator $i$ from a reflexive fuzzy relation $R$ that is $h$ transitive w.r.t. a conjunctor $h$. We have presented both theorems expounding 


\begin{tabular}{c|ccc}
$h \backslash i$ & $T_{\mathbf{L}}$ & $T_{\mathbf{P}}$ & $T_{\mathbf{M}}$ \\
\hline$T_{\mathbf{L}}$ & $T_{\mathbf{L}}$ & $T_{\mathbf{P}}\left(T_{\mathbf{L}}, S_{\mathbf{M}}\right)$ & $T_{\mathbf{L}}\left(T_{\mathbf{L}}, S_{\mathbf{M}}\right)$ \\
$T_{\mathbf{P}}$ & $g_{T_{\mathbf{P}}}^{T_{\mathbf{P}}}$ & $g_{T_{\mathbf{P}}}^{T_{\mathbf{P}}}$ & $g_{T_{\mathbf{P}}}$ \\
$T_{\mathbf{M}}$ & $T_{\mathbf{n M}}$ & $T_{\mathbf{n M}}^{\varphi 1}$ & $T_{\mathbf{M}}$
\end{tabular}

Table 2: Propagation of the transitivity of $R$ to $P$ for the three most important t-norms.

very general results as well as specific propositions treating the most important particular cases.

The general results are of use for any (1-Lipschitz) increasing generator $i$ and any (commutative) conjunctor $h$. These results finally close the study of the transitivity of the symmetric and asymmetric components of a reflexive fuzzy relation. Both the decomposition by means of a generator $i$ and the use of a conjunctor $h$ for describing the transitivity are the most general considerations possible.

The specific results concern the most important particular cases, i.e. those for which both the generator $i$ and the conjunctor $h$ are among the most important t-norms. These particular cases have been a topic of study for many years. However, focus was too often on preservation of transitivity, rather than trying to identify the strongest type of transitivity possible. The specific results provide easy-to-use expressions, except for one case leading to an unwieldy expression.

Despite the non-comprehensive general formulae obtained in Theorems 4.1 and 5.1, we have already proven that these conjunctors have interesting properties in some particular cases [8]. In future work, we intend to study the properties of these conjunctors in more detail.

\section{Acknowledgements}

The research reported on in this paper has been partially supported by Project FEDER-MEC-MTM2007-61193.

\section{References}

[1] Alsina, C. (1985). On a family of connectives for fuzzy sets. Fuzzy Sets and Systems, 16, 231-235.

[2] Alsina, C., Frank, M. J., \& Schweizer, B. (2006). Associative Functions: Triangular Norms and Copulas. World Scientific Publishing Company.

[3] Alsina, C., Nelsen, R.B., \& Schweizer, B. (1993). On the characterization of a class of binary operations on distribution functions. Statist. Probab. Lett., 17, 85-89.

[4] Arrow, K. J. (1951). Social Choice and Individual Values. Wiley. 
[5] Bilgiç, T. (1998). Interval-valued preference structures. European Journal of Operational Research, 105, 162-183.

[6] Dasgupta, M., \& Deb, R. (1996). Transitivity and fuzzy preferences. Social Choice and Welfare, 13, 305-318.

[7] Dasgupta, M., \& Deb, R. (2001). Factoring fuzzy transitivity. Fuzzy Sets and Systems, 118, 489-502.

[8] De Baets, B., De Meyer, H., \& Díaz, S. (2009). On an idempotent transformation of aggregation functions and its application on absolutely continuous Archimedean copulas. Fuzzy Sets and Systems, 160, 733-751.

[9] De Baets, B., \& Fodor, J. (1997). Twenty years of fuzzy preference structures (1978-1997). Belg. J. Oper. Res. Statist. Comput. Sci., 37, 61-82.

[10] De Baets, B., \& Fodor, J. (2003). Additive fuzzy preference structures: the next generation. In: B. De Baets \& J. Fodor (Eds.), Principles of Fuzzy Preference Modelling and Decision Making (pp. 15-25). Academia Press.

[11] De Baets, B., Janssens, S., \& De Meyer, H. (2006). Meta-theorems on inequalities for scalar fuzzy set cardinalities. Fuzzy Sets and Systems, 157, $1463-1476$.

[12] De Baets, B., \& Mesiar, R. (1998). T-partitions. Fuzzy Sets and Systems, 97, 211-223.

[13] De Baets, B., \& Van de Walle, B. (1997). Minimal definitions of classical and fuzzy preference structures. Proceedings of the Annual Meeting of the North American Fuzzy Information Processing Society (pp. 299-304). USA: Syracuse, New York.

[14] De Baets, B., Van De Walle, B., \& Kerre, E. (1995). Fuzzy preference structures without incomparability. Fuzzy Sets and Systems, 76, 333-348.

[15] Díaz, S., De Baets, B., \& Montes, S. (2003). On the transitivity of fuzzy indifference relations. Lecture Notes in Artificial Intelligence, 2715, 87-94.

[16] Díaz, S., De Baets, B., \& Montes, S. (2007). Additive decomposition of fuzzy pre-orders. Fuzzy Sets and Systems, 158, 830-842.

[17] Díaz, S., De Baets, B., \& Montes, S. (2008). On the compositional characterization of complete fuzzy pre-orders. Fuzzy Sets and Systems, 159, 2221-2239.

[18] Díaz, S., Montes, S., \& De Baets, B. (2004). Transitive decomposition of fuzzy preference relations: the case of nilpotent minimum. Kybernetika, 40, 71-88.

[19] Díaz, S., Montes, S., \& De Baets, B. (2007). Transitivity bounds in additive fuzzy preference structures. IEEE Trans. on Fuzzy Systems, 15, 275-286. 
[20] Fernández, E., Navarro, J., \& Duarte, A. (2008). Multicriteria sorting using a valued preference closeness relation. European Journal of Operational Research, 185, 673-686.

[21] Fodor, J., \& Roubens, M. (1994). Valued preference structures. European Journal of Operational Research, 79, 277-286.

[22] Fodor, J., \& Roubens, M. (1994). Fuzzy Preference Modelling and Multicriteria Decision Support. Kluwer Academic Publishers.

[23] Fodor, J., \& Roubens, M. (1995). Structure of transitive valued binary relations. Mathematical Social Sciences, 30, 71-94.

[24] Genest, C., Quesada-Molina, J. J., Rodríguez-Lallena, J. A., \& Sempi, C. (1999). A characterization of quasi-copulas. Journal of Multivariate Analysis, 69, 193-205.

[25] Hájek, P. (1998). Metamathematics of Fuzzy Logic. Dordrecht: Kluwer Academic Publishers.

[26] Haven, E. (2002). Fuzzy interval and semi-orders. European Journal of Operational Research, 139, 302-316.

[27] Herrera-Viedma, E., Herrera, F., Chiclana, F., \& Luque, M. (2004). Some issues on consistency of fuzzy preference relations. European Journal of Operational Research, 154, 98-109.

[28] Janssens, S., De Baets, B., \& De Meyer, H. (2004). Bell-type inequalities for quasi-copulas. Fuzzy Sets and Systems, 148, 263-278.

[29] Klement, E. P., Mesiar, R., \& Pap, E. (2000). Triangular Norms, Kluwer Academic Publishers.

[30] Nelsen, R. (1994). An Introduction to Copulas, Lecture Notes in Statistics, 139. Springer-Verlag.

[31] Ovchinnikov, S. (1991). Similarity relations, fuzzy partitions, and fuzzy orderings. Fuzzy Sets and Systems, 40, 107-126.

[32] Roubens, M., \& Vincke, Ph. (1985). Preference Modelling. Lecture Notes in Economics and Mathematical Systems, 76. Springer-Verlag.

[33] Saminger-Platz, S., De Baets, B., \& De Meyer, H. (2006). On the dominance relation between ordinal sums of conjunctors. Kybernetika, 42, 337350 .

[34] Saminger-Platz, S., De Baets, B., \& De Meyer, H. (2009). Differential inequality conditions for dominance between continuous Archimedean tnorms. Mathematical Inequalities and Applications, 12, 191-208. 
[35] Saminger, S., Mesiar, R., \& Bodenhofer, U. (2002). Domination of aggregation operators and preservation of transitivity. Internat. J. Uncertainty, Fuzziness and Knowledge-Based Systems, 10, 11-35.

[36] Sarkoci, P. (2005). Domination in the families of Frank and Hamacher tnorms. Kybernetika, 41, 349-360.

[37] Van de Walle, B., De Baets, B., \& Kerre, E. (1998). A plea for the use of Eukasiewicz triplets in the definition of fuzzy preference structures. Part 1: General argumentation. Fuzzy Sets and Systems, 97, 349-359.

[38] Van de Walle, B., De Baets, B., \& Kerre, E. (1998). Characterizable fuzzy preference structures. Annals of Operations Research, 80, 105-136. 